\title{
Constructing intersectoral innovation diffusion networks with input-output: how to get relative flows? An illustrative application to six OECD technological systems for the middle ' $90 \mathrm{~s}^{*}$
}

\author{
Sandro Montresor ${ }^{\dagger} \quad$ Giuseppe Vittucci Marzetti ${ }^{\ddagger}$
}

\begin{abstract}
The paper aims at addressing some methodological issues in applying Social Network Analysis indicators to the investigation of nation-wide intersectoral innovation flows matrices. The majority of SNA techniques require dichotomization of the original matrices and suitable relativization procedures, in order to avoid size-biases. The relativization procedures used so far suffer from some limitations, as they either alter the meaning of SNA indicators or do not take into account the composition of countries' final demand.

In order to overcome these limitations, we propose two new different methods and compare them with the existing ones on the basis of their rationale. Rather than with respect to a single cut-off, the comparison is carried out by working out SNA indicators distributions. An illustrative application is carried out by comparing the technological systems of six structurally different OECD countries in the mid-'90s. In so doing, the robustness of their conventional innovation ranking is tested and discussed.
\end{abstract}

${ }^{*}$ The authors gratefully acknowledge comments and suggestions from the participants to the 16th International Input-Output Conference (Istanbul, July 2-6, 2007) on a previous version of this paper. The usual caveat applies. Giuseppe Vittucci Marzetti gratefully acknowledges the financial support from the Autonomous Province of Trento (Grants 2006).

${ }^{\dagger}$ Department of Economics, Univ. of Bologna, Italy. E-mail: sandro.montresor@unibo.it

${ }^{\ddagger}$ Dpt. of Economics, Univ. of Trento, Italy. E-mail: giuseppe.vittucci@economia.unitn.it 
Keywords Technological systems; Embodied innovation flows; Input-Output analysis; Network analysis

JEL Classification: O33; O38; O39

\section{Introduction}

Although it gained popularity in the early '90s, the system analysis of the innovative process has recently undergone an important transformation. The need of complementing qualitative empirical analyses of those institutions and organizations which make up systems of innovation - national, sub-national, super-national and regional - with rigorous quantitative measurements and evaluations of their relationships, structure and performance, has spurred to look for new and more suitable methodological tools.

Among these, as Christian DeBresson shew so brightly with his long research career, the analysis of "economic interdependence" represents an extremely powerful tool of investigation (DeBresson, 1996a). Indeed, combining input-output analysis with that of innovation activity, and building up intersectoral innovation flows matrices, provides the researcher with a precious 'map' trough which to investigate the constitutive relationships of a system of innovation. What is more, this map can be fruitfully explored by choosing a suitable "navigator", that is network analysis. Sectors and intersectoral flows can be in fact dealt with as the constitutive elements of innovation networks whose properties can be investigated through Social Network Analysis (SNA). As has been shown by a number of studies (see, for instance, DeBresson, 1996a; Leoncini and Montresor, 2003b; Chang and Shih, 2005; Montresor and Vittucci Marzetti, 2007b), several network analysis indicators have an immediate and equivalent meaning in the system analysis of innovation.

With respect to this research program, the present paper represents a sort of step behind, rather than ahead. Its intention is in fact that of going back to those "crucial" methodological issues one has (as we had!) to tackle to obtain sound empirical results. In particular, we intend to "lift the carpet" and 
take "frankly" the problem of scale-effects in comparing different technological systems at the country level on the basis of their innovation intersectoral flows matrices. In this vein, the paper might sound excessively technical but, hopefully, also show how pure (and noisy) technicalities are responsible for larger (and attractive) techno-economic results. ${ }^{1}$

The structure of the paper is the following. Section 2 sketches the theoretical background of the paper. Section 3 discusses the scale issue and the problem of obtaining proper relative intersectoral innovation flows to get rid of it. Section 4 critically reviews previously proposed relativization procedures and compares them with some new methodological proposals. Section 5 sets these procedures at work in a purely illustrative application carried out by comparing the technological systems of 6 structurally different OECD countries (Japan, Korea, Netherlands, Poland, Spain, USA) in the middle '90s, and tries to get signs of systematic vs. occasional differences. Section 6 concludes.

\section{Theoretical background: investigating technological systems through intersectoral network analysis}

The methodological issue this paper addresses is typically faced in comparing the technological systems of two or more countries (or sectors) by looking at their intersectoral innovation flows. ${ }^{2}$

This research program, which has recently attracted a number of studies (see, for instance, DeBresson, 1996a; Leoncini and Montresor, 2003b; Chang and Shih, 2005), draws on the following theoretical background.

\footnotetext{
${ }^{1}$ In this last respect, the reader will be disappointed. Not so much techno-economic results here! For consolation, see, for example Montresor and Vittucci Marzetti (2007b).

${ }^{2}$ More in general, the same issue is relevant whenever two or more countries are compared on the basis of a certain intersectoral matrix which distributes the sectoral values of a certain variable along their subsystem structure. The comparative analysis of de-industrialization and tertiarisation processes is thus another potential field of application for it (Montresor and Vittucci Marzetti, 2007a).
} 
First, the innovative process does not occur among (atomistic) economic agents acting in isolation. Rather, it is innervated and shaped by a set of relationships through which innovative institutions and organizations (e.g. firms, research labs, universities, patent offices, and the like) interact, learn and originate different kinds of systems (of innovations) (Lundvall, 1992; Edquist, 1997; Edquist and McKelvey, 2000).

Second, not only are innovative relationships institutional and affected by the topographical, geographical space in which they operate. But they are also technical and economic in their nature, and thus also affected by "an abstract economic space of supply and demand of different goods (as represented by input-output matrices) and [a] technical space (as represented by the techno-functional classification of patents)" (DeBresson, 1996b, p.151). Accordingly, a broad notion of Technological System (TS) (Leoncini, 1998; Leoncini and Montresor, 2003a) is required in order to retain the complexity of the innovative process.

Third, the constitutive relationships of a TS can be proxied and mapped by building up a suitable matrix of intersectoral innovation flows, namely, a $(n \times n)$ matrix of $\mathrm{R} \& \mathrm{D}$ flows embodied in vertically integrated subsystems such as the following:

$$
\mathbf{R}=\hat{\mathbf{r}} \hat{\mathbf{q}}^{-1}(\mathbf{I}-\mathbf{A})^{-1} \hat{\mathbf{y}}=\hat{\mathbf{r}} \mathbf{B}
$$

where $\hat{\mathbf{r}}, \hat{\mathbf{q}}$, and $\hat{\mathbf{y}}$ are the sectoral diagonal vectors of, respectively, $R \& D$ expenditures, gross production and final demand, and $(\mathbf{I}-\mathbf{A})^{-1}$ is the Leontief inverse based on the domestic input-output coefficients matrix (A) (e.g. Marengo and Sterlacchini, 1990). Indeed, given the way it is defined, such a matrix actually reflects the functioning of both the innovative sub-system (proxied by R\&D expenditure) and the production sub-system (proxied by input-output tables) of a TS. Furthermore, the resort to the operator $\mathbf{B}$ in reclassifying $\mathrm{R} \& \mathrm{D}$ flows from pure sectors into vertically integrated sectors (or subsystems) allows the same flows to retain also the market side of a TS 
through the role of final demand. ${ }^{3,4}$

Fourth, the structure of a TS and the different role of its constitutive sectors can be analyzed, especially in a cross-country comparative framework, by applying to intersectoral innovation matrices suitable Social Network Analysis (SNA) techniques and indicators (Leoncini et al., 1996; Leoncini and Montresor, 2000a,b). Indeed, the economic sectors and the intersectoral techno-economic flows of these matrices can be envisaged as, respectively, the vertices (or nodes) and the arcs of the constitutive network of the correspondent TS. In particular, three SNA indicators are particularly useful in comparing different TSs. ${ }^{5}$

The first one refers to the TS as a whole, and is the density $(\delta)$ of the correspondent network of $n$ sectors, defined as:

$$
\delta(t)=\frac{\sum_{i} \sum_{j(i \neq j)} d_{i j}(t)}{n(n-1)} \text { with } 0 \leq \delta(t) \leq 1
$$

where $d_{i j} \in\{0,1\}$ is the element of the so called "contingency matrix" $\mathbf{D}(t)$, amounting to the dichotomic transformation - according to a certain cut-off $t$ - of the matrix $\mathbf{R}$ or, as we will argue in the next section, of a suitable relative transformation of it (an issue on which we will return later). The techno-economic interpretation of $\delta(t)$ with respect to a TS is quite immediate: the larger (the smaller) is $\delta(t)$, the more (the less) dense is the network, the more (the less) connected (i.e. systemic) is the correspondent TS.

Unlike the previous one, the second SNA indicator refers to each vertex $j$ of a network, and is given by its degree, or more precisely, indegree and outdegree centrality. This indicator is nothing but the number of vertices through which

\footnotetext{
${ }^{3}$ The B operator was first proposed by Siniscalco (1982). Each row of it adds up to 1 and shows "the shares of output of each sector which contribute to the different subsystems". Thus, B can be used to reclassify any physical or value magnitude from sectors into subsystems. As noted by Rampa (1982), it is relative price invariant and depends not only on strict technological factors, but also on the structure of final demand.

${ }^{4}$ As has been argued elsewhere (Leoncini and Montresor, 2005), the map can be refined by considering, still in an input-output framework, the role of the foreign market and of the institutional set-up.

${ }^{5}$ As Montresor and Vittucci Marzetti (2007b) show, the same kind of analysis can be fruitfully employed also to detected and investigate different forms of innovation clusters within the TSs.
} 
a certain node $j$ is reached by (indegree) and reaches (outdegree) the other $n-1$ vertices of the network. In a directed graph, such as that correspondent to the intersectoral innovation matrices we are dealing with, the indegree and outdegree centrality of a vertex $j$ are distinct and formally defined as follows:

$$
\begin{gathered}
C_{j}^{\text {in }}(t)=\sum_{i(i \neq j)} d_{i j}(t) \quad \text { with } 0 \leq C_{j}^{\text {in }}(t) \leq n-1 \\
C_{j}^{\text {out }}(t)=\sum_{j(j \neq i)} d_{i j}(t) \quad \text { with } 0 \leq C_{j}^{\text {out }}(t) \leq n-1
\end{gathered}
$$

where, as before, $t$ refers to a certain cut-off value. When dealing with TSs, given that inward and outward arcs represent, respectively, intersectoral innovative acquisitions and diffusions, the two measures of centrality can help determining the degree of dependency or pervasiveness of the sectors in a TS.

The last SNA indicator we consider refers to the TS as a whole again, and is given by the degree, or more precisely, indegree and outdegree centralization of the correspondent network, defined as:

$$
\begin{gathered}
H^{\text {in }}(t)=\frac{\sum_{j}\left(C_{j^{*}}^{\text {in }}(t)-C_{j}^{\text {in }}(t)\right)}{(n-1)^{2}} \text { with } 0 \leq H^{\text {in }}(t) \leq 1 \\
H^{\text {out }}(t)=\frac{\sum_{j}\left(C_{j^{*}}^{\text {out }}(t)-C_{j}^{\text {out }}(t)\right)}{(n-1)^{2}} \text { with } 0 \leq H^{\text {out }}(t) \leq 1
\end{gathered}
$$

where $C_{j^{*}}^{i n}$ and $C_{j^{*}}^{\text {out }}$ are, respectively, the indegree and outdegree centrality of the most central vertex $j^{*}$.

As low values of the centralization identifies a network with similar (centrality) positions, the correspondent TS can be deemed one in which its sectoral partitions are "evenly distributed". Conversely, large centralization values refer to TSs with highly "hierarchic" sectoral partitions (Leoncini and Montresor, 2000b).

\section{3 "Scale" and "cut-off" in comparing different TSs: two delicate issues}

Although the interpretative power of a network (intersectoral) analysis of different TSs is quite high, this can be obtained only by dealing satisfactorily 
with two crucial methodological problems.

The first problem is due to the fact that the SNA indicators described above require us to work with dichotomic matrices, that is matrices made up of $1 s$ and $0 s$ depending on the values of the retained matrices of intersectoral innovation flows being, respectively, larger and smaller than a certain cut-off value $t$. Although quite crucial, the choice of this cut-off value, and the sensitivity of the SNA results on it, is insufficiently addressed by the network analyses of techno-economic phenomena.

In order to attenuate this problem, Leoncini and Montresor (2000b) started suggesting to work with more cut-off values through a two-step procedure: (i) for each of the $z$ TSs to be compared, building up a density distribution $\mathbf{d}\left(\mathbf{t}_{z^{*}}\right)$ using as cut-off values the ordered distribution, $\mathbf{t}_{z^{*}}$, of the $n \times(n-1)$ cells of a proper reference matrix, that is of a reference TS, $z^{*}$; (ii) by comparing the $z$ density distributions, extracting "heuristically" some cut-off values out of the distribution $\mathbf{t}_{z^{*}}$, with respect to which carrying out the rest of the network analysis and checking for their robustness. In a recent paper, Montresor and Vittucci Marzetti (2007b) refined this procedure by suggesting to run step (i) with respect to a "super-vector" of cut-offs $\left(\mathbf{t}_{Z}\right)$, whose $Z$ elements are obtained by ordering the cells of all the $z$ matrices to be compared $(Z=(n(n-1)) \times z)$. In spite of the difficulties in getting general density results in front of such numerous cut-off values, this procedure reveals superior for a set of reasons for which the interested reader should refer to the relative paper.

The second crucial issue is even preliminary to the first one, and refers to the choice of the proper "relative" intersectoral innovation flows matrix to which the dichotomization and the network analysis should be applied. Indeed, although the need of transforming the absolute values of the $\mathbf{R}$ matrices through which the correspondent TSs are compared - into relatives ones might appear intuitive, the actual way such relative matrices are obtained is far from innocuous: conversely, as we intend to show with this paper for the first time, the results of a network intersectoral analysis of the TSs are quite sensitive to it.

First of all, given the way $\mathbf{R}$ is defined, it is straightforward that applying the SNA indicators directly to the $\mathbf{R}$ matrices of the $z$ TSs to be compared - of 
course, after having expressed the correspondent vectors of $R \& D$ expenditure in PPP terms - will end out by yielding misleading results. Should country 1 be much larger (in economic terms) than country 2, and thus have a larger scale of R\&D activities across all the considered sectors, the density analysis would show, a fortiori, higher values for the former than for the latter country with respect to all the cut-offs. However, concluding that TS1 is more connected than TS2 would be, in this case, not guaranteed: although less consistent, and thus systematically excluded from the dichotomization, the embodied sectoral R\&D flows of the latter might be more diffusely distributed than those of the former. Actually, they could be more polarized (i.e. less systemic), but by using absolute R\&D flows as cut-offs values we are unable to capture it.

In order to get rid of scale effects, and make density and the other SNA indicators more robust in informing about the relational structure of the compared TSs, we need to normalize the absolute intersectoral flows of $\mathbf{R}$ in some way. But with respect to what? Indeed, scale differences across different TSs could be traced at different levels. Not only could the scale of R\&D activities of different TSs be systematically different, but two TSs could have similar R\&D scales and, still, systematically different demand volumes - spurring differently the intersectoral innovation flows in a sub-system framework - or different production volumes - conveying differently the intersectoral innovation flows embodied in them. What is more, considering that TSs have also a sectoral dimension, along with a national one (Malerba, 2004), there could be systematic scale differences across different subsystems. In the light of these considerations, rather than one relativization procedure only, different ways of getting normalized intersectoral flows should be considered. As we will show in the following, these different procedures yield different results, so that their choice should be based on an accurate evaluation of their pros and cons and, above all, of their rationale.

\section{Alternative relativization procedures}

Although the outcome is always represented by some kind of relative matrix, different relativization procedures can be accomplished, depending on the scale 
of operation one wants to get rid of and on the national or sector/subsystem focus of the analysis.

\subsection{Unit value matrix}

A first possible relativization procedure is that proposed by Chang and Shih (2005), who suggest to compare the intersectoral structure of different TSs by resorting to a unit value matrix $\left(\mathbf{R}^{\text {unit }}\right)$ defined as follows: ${ }^{6}$

$$
\mathbf{R}^{\text {unit }}=\hat{\mathbf{r}} \hat{\mathbf{q}}^{-1}(\mathbf{I}-\mathbf{A})^{-1}
$$

Let us observe that using this matrix amounts to dividing each column of the original matrix $\mathbf{R}$ by the final demand level of the correspondent subsystem, that is:

$$
\mathbf{R}^{\text {unit }}=\mathbf{R} \hat{\mathbf{y}}^{-1}
$$

Accordingly, we can conclude that the different subsystems of each TS are in this way scaled down to the same unit final output, irrespectively of the ratios between the original operational scales of the different subsystems, which could be different in different TSs.

In the light of this latter fact, the use of $\mathbf{R}^{\text {unit }}$ entails a focus on the individual subsystems of each TS. Therefore, this procedure might be justified when one is interested in the TS conceived as a distinguishable "constellation" of individual subsystems. On the other hand, let us observe that $\mathbf{R}^{\text {unit }}$, while it gets rid of the differences in countries' overall GDP - which is desirable - it also neutralizes countries' differences in its structure - which is not as desirable. $^{7}$

\footnotetext{
${ }^{6}$ Although Chang and Shih (2005) convert all the values in US dollars, this does not prove strictly necessary. Indeed, denoting with $E$ the nominal exchange rate of the home currency to the US dollar, we have that the matrix obtained by using $E\left(\mathbf{R}_{E}^{\text {unit }}\right)$ is equivalent to that without $\left(\mathbf{R}^{\text {unit }}\right)$ :

$$
\mathbf{R}^{\text {unit }}=\hat{\mathbf{r}} \hat{\mathbf{q}}^{-1}(\mathbf{I}-\mathbf{A})^{-1}=E^{-1} \hat{\mathbf{r}} \hat{\mathbf{q}}^{-1} E\left(\mathbf{I}-E^{-1} \mathbf{A} E\right)^{-1}=\mathbf{R}_{E}^{\text {unit }}
$$

${ }^{7}$ It is also worth noting that such method tends to underestimate the weight of the $R \& D$ efforts of the less developed countries, unless a PPP correction is introduced.
} 


\subsection{Unit basket of final demand}

In order to retain the different structure of the final demand in the compared TSs, while still getting rid of overall scale differences, we suggest to resort to a different relativization procedure, and work out the following matrix:

$$
\mathbf{R}^{\text {basket }}=\frac{1}{\mathbf{i}^{\prime} \mathbf{y}} \mathbf{R}
$$

where $\mathbf{i}^{\prime}$ is a unit row vector.

The matrix $\mathbf{R}^{\text {basket }}$ calculates the innovation flows embodied in the intermediate production ones "activated" by a unit basket of final demand. Thus, while it still scales down the different subsystems to a comparable cross-country level, it leaves unaltered the operational scale ratios between the different subsystems of each TS. Just to give an example, in the case of a fictitious economy made up of three sectors, whose final demand vector is $\mathbf{y}=(1500,2500,1000)$, the correspondent unit basket of final demand will be $(0.3,0.5,0.2)$, unlike $(1,1,1)$ as for $\mathbf{R}^{\text {unit }}$.

\subsection{Normalized $\mathrm{R}$}

Although the previous relativization removes cross-country differences connected with the "size" of their economy, their relative ranking in terms of aggregate $R \& D / G D P$ ratios still affects the network analysis which is based on it.

If one is interested in focusing on the purely relational characteristics of the different TSs, that is, on how embodied innovation flows are distributed among the different subsystems within each TS, irrespectively of the value of such flows as a proportion of the overall size of the system, he needs to move to a different relativization. In this case, a possible relativization procedure we suggest could be the simple normalization to one of the $\mathbf{R}$ matrices, that is:

$$
\mathbf{R}^{n o r m}=\frac{1}{\mathbf{i}^{\prime} \mathbf{R} \mathbf{i}} \mathbf{R}=\frac{1}{\mathbf{i}^{\prime} \mathbf{r}} \mathbf{R}
$$

Indeed, the ratio between each element of $\mathbf{R}^{\text {basket }}\left(r_{i j}^{\text {basket}}\right)$ and the correspondent element of $\mathbf{R}^{\text {norm }}$ ( $r_{i j}^{\text {norm }}$ ) is constant and equal to the country's aggregate 
$R \& D / G D P$ ratio:

$$
\frac{r_{i j}^{\text {basket }}}{r_{i j}^{\text {norm }}}=\frac{r_{i j}}{\mathbf{i}^{\prime} \mathbf{y}} / \frac{r_{i j}}{\mathbf{i}^{\prime} \mathbf{r}}=\frac{\mathbf{i}^{\prime} \mathbf{r}}{\mathbf{i}^{\prime} \mathbf{y}} \quad(i, j=1,2, \ldots, n)
$$

\subsection{Matrix C}

A last possible method to relativize the matrix $\mathbf{R}$ is that of normalizing the subsystem innovative acquisitions, that is of dividing each cell of the original matrix by the sum of the correspondent column, thus building up a matrix C defined as follows:

$$
\mathbf{C}=\mathbf{R}\left(\widehat{\mathbf{i}^{\prime} \mathbf{R}}\right)^{-1}
$$

where the hat symbol is used to denote diagonalization. ${ }^{8}$

This matrix, put forward by Leoncini et al. (1996), has been used by Leoncini and Montresor (2000b, 2003b, 2005) and, more recently, by Montresor and Vittucci Marzetti (2007b) for is "mixed" properties. On the one hand, like $\mathbf{R}^{\text {norm }}$, $\mathbf{C}$ emphasizes "pure" relational aspects. On the other hand, unlike $\mathbf{R}^{\text {norm }}$, but like $\mathbf{R}^{\text {unit }}$, the focus is kept on the individual subsystems of each TS.

\subsection{A synthesis}

In brief, the four relativization procedures described above combine two different levels of analysis:

(i) the first level concerns the dimension along which one might want to get rid of scale effects, that is, economic activity (proxied by the volume of final demand) or technological activity (proxied by R\&D expenditure);

(ii) the second dimension refers instead to the focus of the analysis one wants to carry out through relative matrices, that is, system-focus or subsystem-focus (see Table 1)

\footnotetext{
${ }^{8}$ It is worth noting that, given the way $\mathbf{R}$ is defined, dividing its cells by the sum by row simply returns the operator $\mathbf{B}$; in formal terms:

$$
(\widehat{\mathbf{R i}})^{-1} \mathbf{R}=(\widehat{\mathbf{r}} \widehat{\mathbf{B}} \mathbf{i})^{-1} \hat{\mathbf{r}} \mathbf{B}=(\widehat{\mathbf{r} \mathbf{i}})^{-1} \hat{\mathbf{r}} \mathbf{B}=\hat{\mathbf{r}}^{-1} \hat{\mathbf{r}} \mathbf{B}=\mathbf{B}
$$
}


Table 1: Rationale of the relativization procedures

\begin{tabular}{llll}
\hline & & \multicolumn{2}{l}{ Focus of the analysis } \\
\cline { 2 - 3 } & & System & Subsystem \\
\cline { 2 - 3 } Relativization dimension & Economic & $\mathbf{R}^{\text {basket }}$ & $\mathbf{R}^{\text {unit }}$ \\
& Technological & $\mathbf{R}^{\text {norm }}$ & $\mathbf{C}$ \\
\hline
\end{tabular}

On principle, $\mathbf{R}^{\text {basket }}$ and $\mathbf{R}^{\text {norm }}$ appear more consistent with the inner logic of the TS analysis. However, looking the TS as an "artificial constellation" of subsystems might be preferable when one is interested in recovering, also and above all, their different centrality in different TSs. As we will argue in the following, the choice of one of the four procedures should be inspired by the research questions one intends to address. Thus, there is no one absolute best, or worst, procedure among them.

\section{An illustrative application}

In order to fully grasp the differences among the four relativization procedures sketched in the previous section, by using input-output data taken from the "new" OECD Input-Output Database (2005) and crossing them with data on sectoral R\&D expenditure (OECD ANBERD Database, 2005), we have worked out the $\mathbf{R}$ matrix (Equation (1)) with respect to 6 OECD countries, chosen out of 15 available countries for exemplification purposes: Japan, Korea, Netherlands, Poland, Spain and the USA. ${ }^{9}$

It should be stressed that the application is meant to be purely illustrative. In other words, we will deliberately refrain from using the application results to enlarge our knowledge about these 6 TSs, although, to be sure, in some cases we could not avoid it.

With respect to these TSs (i.e. $\mathbf{R}$ matrices), the four relativization procedures have been used to obtained four different benchmarks, with

\footnotetext{
${ }^{9}$ Although matrix inversions have been carried out for each country at the maximum level of disaggregation in order to reduce the distortions introduced by sectoral aggregation, the blanks in the series of the latter dataset have forced us to limit our empirical application to 16 sectors only (see Appendix A for details).
} 
respect to which we have then applied the dichotomization method discussed in Section 3. In other words, for each relativization procedure, each TS has been transformed into a series of 1440 dichotomized matrices $(\mathbf{D}(t))$, one for each element of the "super-vector" of cut-offs $\left(\mathbf{t}_{Z}\right)^{10}$, and with respect to these matrices the TS distributions obtained by applying the network indicators discussed in Section 2 have been worked out.

In so doing, it is therefore possible to analyze how the different network indicators are distributed for each relativization procedure.

\subsection{Density distributions}

To start with, let us consider how the relative distribution of the six TSs changes in moving from one relativization method to the other when their densities (Equation (2)) are considered (Figure 1). By applying the first method (Section 4.1), Japan actually appears the densest TS all along the range of cut-off, followed by the USA, Korea and, at a distant, Netherlands, Spain and Poland (Figure 1(a)). There is practically no inversion in this ranking and one can therefore conclude that, no matter which is the chosen cut-off, Japan is at least as dense as all the other countries in the sample, thus, the most connected TS among the chosen ones. On the contrary, Poland is the least connected TS. ${ }^{11}$

However, when the structure of the final demand is considered, that is, by applying the second method (Section 4.2), the ranking of the densest TSs becomes more blurred and Korea jumps first over a quite wide range of cut-off values (Figure 1(b)). Thus, taking into account the proportion in which the different subsystems are actually operated within each country, thus properly retaining also the market sub-system in the TS characterization, is by no means neutral. ${ }^{12}$

\footnotetext{
${ }^{10}$ As we have said, the $\mathrm{Z}$ elements of $\mathbf{t}_{Z}$ are obtained by ordering the cells of all the 6 relativized matrices, so that $Z=(15 \times 16) \times 6=1440$.

${ }^{11}$ At first sight, this was the picture one could expect by thinking of what we know about these TSs in qualitative and quantitative terms. See, for example, Leoncini and Montresor (2003b) and OECD (2003).

${ }^{12}$ The substantial improvement of Korea's position with the second method might also be partly due to the underestimation of the contribution of R\&D efforts for developing
} 
By looking at the previous density distributions, one is legitimated to suspect that the low ranking of Poland and Spain, although after having discounted the different scale of their economy, is still due to the low aggregate R\&D/GDP ratios compared to the others. Discounting for this further aggregate factor in TSs' comparisons and confronting the different TSs on the ground of their "pure" relational aspects is however possible. The density distributions of Figure 1(c), obtained by working with normalized $\mathbf{R}$ matrices (Section 4.3), actually show that this can radically change the overall picture. Indeed, quite surprisingly, now Poland and Spain rank, respectively, first and second all along the range, while the least connected TS becomes Netherlands. This means that the former two TSs, once discounted for their relatively low level of aggregate R\&D expenditure, turn out to be in fact highly connected. On the contrary, in the Netherlands, also neglecting the quite low R\&D/GDP ratio characterizing such country with respect to Japan, Korea and the USA, the TS turns out to be only weakly connected, so to say "structurally".

As for as $\mathbf{R}^{\text {norm }}$ is concerned, it is worth emphasizing that, although the sum of its elements is equal to one by definition, that of the elements out of the main diagonal can range from 0 to 1 . Thus, the TS density distributions worked out from this matrix actually results from: on the one side, the weight of the intersectoral embodied innovation flows on the intrasectoral ones; on the other side, the distribution of such intersectoral flows among the different sectors. As shown by Figure 2, for all the six analyzed countries but Japan, the ranking in terms of weight of intersectoral innovation flows over intrasectoral ones corresponds to that derived from the analysis of countries' density distributions. ${ }^{13}$ Japan is instead an exception suggesting that, although intersectoral flows are quite high in absolute terms (23.4\% of the total), they are apparently more polarized in relative terms, thus leading to the low ranking of the correspondent TS in Figure 1(c). The simple comparison of the distribution of the extradiagonal values for Japan

countries introduced by the former method if no PPP correction is used. And this bias should be taken into account when economic systems with different levels of development are compared. For the delicate issue of R\&D data on PPP see also Dougherty et al. (2007).

${ }^{13}$ In passing, let us note that intersectoral embodied flows are quite low compared to the intrasectoral ones, amounting on average for the six countries to $18 \%$ of the total flows, with a coefficient of variation of $32.6 \%$. 
and Spain confirms, in terms of basic indicators, this result (Figure 3).

To conclude, Figure 1(d) shows the density distributions obtained by using the matrix $\mathbf{C}$ of normalized subsystem innovative acquisitions (Section 4.4). This time, instead of being relativized with respect to the total innovation flows of the correspondent TS, the inward flows of each subsystem are normalized with respect to its own total acquisitions. Thus, each subsystem is treated in relative isolation. Accordingly, what can in fact affect the density distributions of each TS as a whole is not as much the ratio between its intrasectoral and intersectoral total embodied innovation flows, as in the former method. But rather the weight of the innovative acquisitions each subsystem gets from the other sectors on the total inward flows of the subsystem itself.

Figure 4 reports the box-plots of the distributions of these weights for the different TSs and shows that the TS density distributions of Figure 1(d) can be partly explained by the distribution of such weights. For example, the most (least) structurally dense TS, that is Poland (Netherlands), actually shows the highest (lowest) ordered statistics when compared with the others. ${ }^{14}$

By using $\mathbf{C}$, the general picture provided by Figure 1(c) gets somewhat confirmed, while the TS density distributions become more similar. However, it has to be emphasized that, given the main subsystem focus of this method, when one wants to derive features related to the overall TS, as something different from the sum of its subsystems, relativizing $\mathbf{R}$ matrices with such procedure turns out to be not fully appropriate. ${ }^{15}$

\footnotetext{
${ }^{14}$ However, it is worth noting that, assuming normality - not rejected at the $10 \%$ significance level ( $5 \%$ for Netherlands) by the Kolmogorov-Smirnov test - and variance homogeneity across the different groups - not rejected by the Levene statistic $(\mathrm{F}(5,90)=.244$, p-value $=.942)-$, the ANOVA test does not reject the null hypothesis of mean equality of these weights across the different TSs $(\mathrm{F}(5,90)=.354, \mathrm{p}$-value $=.88)$, and this result does not change with non parametric statistics (Kruskal Wallis test p-value $=.794$ ).

${ }^{15}$ In this respect, Chang and Shih (2005) are right when they point out that this relativization procedure is "unable to produce a comparable base for displaying the differences between [...] countries" (2005, p.157), although it has to be stressed that, as seen before, such remark can be equally applied to their method too.
} 


\subsection{Centralization distributions}

Not only do the relativization procedures discussed above affect density, but also the other network indicators. This is particularly true when their impact on the indegree and outdegree centralization (see Section 2) distributions is considered. In particular, as shown by Figures 5 and 6 , the relativization procedures affect the absolute and local maximums of these indicators as well as the relative ranking of the TSs according to them. ${ }^{16}$

At the outset, it should be noted that, if the density distributions are quite different - such as when one deals with TSs showing remarkable differences in their aggregate $R \& D / G D P$ ratio - by applying one of the first two relativization methods (Figures $1(\mathrm{a})$ and $1(\mathrm{~b})$ ) the relative degree centralization distributions are not "centered", and this can make cross-TS comparisons in terms of centralization very sensible to the cut-off which is actually chosen (Figures 5(a), 5(b), 6(a) and 6(b)). In this case, rather than comparing the TS centralization at the same cut-off, as for the density analysis, a sounder choice would be to compare the absolute and local maximums of the indicator for the different TSs. In other words, in the present case, centralization would serve different research questions: irrespectively of the chosen threshold flow-size (i.e. cut-off), which might be different in the different TS to be compared, which is the maximum value of degree centralization attained by each TS? Which is the relative differences in such maximums? For which flow-size difference with respect to such maximum is a certain TS equally hierarchical from another one?

Although this holds true for both $\mathbf{R}^{\text {unit }}$ and $\mathbf{R}^{\text {basket }}$, which of the two is actually chosen could be decided on the ground of their inner logic (Section 4.5): when the indicator refers to the TSs as a whole, as degree centralization does, and the latter are not simply seen as a constellation of individual subsystems, the second method should be preferred (Figures 5(c) and 6(c)).

As far as the third relativization procedure is concerned, that is $\mathbf{R}^{\text {norm }}$, an interesting relationship with $\mathbf{R}^{\text {basket }}$ should be noticed. Given that, for each TS, the ratio between every element of $\mathbf{R}^{\text {basket }}$ and the correspondent

\footnotetext{
${ }^{16}$ Given the way it is defined, the degree centralization, inward or outward, tends to 0 when the density of the correspondent network tends to 1 or 0 .
} 
element of $\mathbf{R}^{\text {norm }}$ is constant and equal to country's aggregate $R \& D / G D P$ ratio (Equation (11)), applying the latter procedure does not alter the centralization ranking of absolute and local maximums worked out with the former, but simply "centralizes" the TSs's distributions. As this makes the TSs more easily comparable (Figures 5(c) and 6(c)) in terms of centralization, and does not affect the centralization values, when the latter are the focus (irrespectively of the correspondent cut-off) $\mathbf{R}^{\text {norm }}$ should thus be preferred.

Following the same line of reasoning and with the same research question in mind, one can argue that this method should be preferred also to the last one (Figures $5(\mathrm{~d})$ and $6(\mathrm{~d})$ ). Indeed, although by using the matrix $\mathbf{C}$ one can still obtain "centralized" distributions, the main focus of the method on the individual subsystems should prevent its application when the analysis is mainly concerned with the whole TSs.

A last comment should be spent on the TS centralization distributions when indegree rather than outdegree centralization indicators are used. By comparing Figure 5 and 6 it appears immediately that, in spite of the same relativization arguments, the TS rankings in the two cases are different. Just to make an example, when outdegree centralization is considered, the Netherlands emerge for the highest values and this, apart from the low density of this TS, seemingly reveals a high dependency on few key sectors for innovation diffusion. Conversely, by looking at indegree centralization, it is Japan which stands out, revealing the presence in its TS of one or more sectors which are dependent on the rest of the TS much more than the average.

More in general, the different meaning of indegree and outdegree centralization should be clearly disentangled, whatever relativization procedure is adopted.

\subsection{Centrality distributions}

Given the close relationship with the correspondent centralization indicators, the impact of the alternative relativization procedures on the last network indicator presented in Section 2, that is, degree centrality, does not require much further exploration. For exemplification purposes only, we have chosen textiles (4) out of the 16 available subsystems we have for each TS (Appendix 
A) and decided to accomplish the analysis only with respect to the indegree centrality (Figure 7).

At the outset, we should notice that, although this sector is structurally dependent in technological terms, its indegree centrality is quite different across the six TSs for a number of cut-offs. What is more important, the relativization procedure crucially alters the centrality gap of the sector across the TSs, so that the relative choice has to be carefully thought also in this last respect.

Following the criteria set in Section 4, the relativization methods more suitable for the analysis of degree centrality should be the first $\left(\mathbf{R}^{\text {unit }}\right)$ and the last one $(\mathbf{C})$. Indeed, we are now comparing a specific subsystem in different TSs, thus, the relativization procedure should be able to discount for all the factors more related to the TS it belongs to, rather than to the subsystem itself. $^{17}$

In so doing, it is possible just to rescale the subsystem to the same operational scale in all the TSs (Figure $7(\mathrm{a})$ ) or normalizing to one its total embodied innovation inflows (Figure $7(\mathrm{~d})$ ).

\section{Conclusions}

Although the problem of dichotomizing the original value matrices is usually retained the most crucial in applying the majority of the tools of network analysis - which cannot be directly applied to value graphs - that of getting "suitable" matrices to dichotomize is no less crucial. ${ }^{18}$ As we have argued in this paper, when network analysis is applied to compare, across different countries, input-output based matrices which 'distribute' across the sectors and/or sub-systems of an economy some techno-economic kind of variable, dichotomization has to be preceded by a suitable relativization. Indeed, in

\footnotetext{
${ }^{17}$ It has to be stressed that the situation would be different if one instead had to compare two or more subsystems within the same TS in order to infer their relative position in it.

${ }^{18}$ In the present paper the dichotomization issue has been dealt with only marginally, but it is a really crucial one to which we have dedicated at more length elsewhere (see, for example Montresor and Vittucci Marzetti (2007b)). Still, the paper incorporates the results we have obtained in these other works.
} 
the absence of this relativization, the application of network analysis would be affected by size-biases and lead to misleading results.

Although referred to the combined use of input-output and network analysis in comparing different Technological Systems (TSs), the methodological issue we tackle in the paper is thus quite general, as could arise also in other application contexts. Similarly, the two new relativization procedures we suggest applying to "our" intersectoral innovation flows of embodied R\&D - that is, (a) scaling down the different subsystems to a comparable cross-country level, but leaving unaltered the operational scale ratios between the different subsystems ( $\mathbf{R}^{\text {basket }}$ ); (b) normalizing to one the original matrix $\left(\mathbf{R}^{\text {norm }}\right)$ along with the other 'standard' two with which we compare them - (c) scaling down to the same unit final output the different subsystems within each TS ( $\left.\mathbf{R}^{\text {unit }}\right)$; (d) building up the matrix of normalized innovative acquisitions within each subsystems $(\mathbf{C})$ - are based on a rationale which could be more generally extended. And the same holds true for the rationale of their choice, which should cross the system (methods $\mathbf{R}^{\text {basket }}$ and $\mathbf{R}^{\text {norm }}$ ) or subsystem (methods $\mathbf{R}^{\text {unit }}$ and $\mathbf{C}$ ) focus of the analysis, with the economic (methods $\mathbf{R}^{\text {unit }}$ and $\mathbf{R}^{\text {basket}}$ ) or technological (methods $\mathbf{R}^{\text {norm }}$ and $\mathbf{C}$ ) dimension one wants to normalize.

The illustrative application we have carried out with respect to 6 (arbitrarily chosen) OECD countries in the middle '90s clearly shows that such a choice of relativization does matter. When the relative $\mathbf{R}$ matrices are used to obtain the network-map of the correspondent TSs - through the relative contingency matrices - and the system-connectivity, hierarchical structure and sectoral dependency/pervasiveness of these TSs are worked out - by means of, respectively, density, degree centralization and degree centrality - their rankings are extremely sensitive to the adopted relativization procedure. For example, as far as density is concerned, Japan and Poland appear, respectively, the most and the least dense/systemic TS among the 6 only when $\mathbf{R}^{\text {unit }}$ is used. Conversely, when the structure of the final demand is considered, that is, by applying $\mathbf{R}^{\text {basket}}$, and taking into account the proportion in which the different subsystems are actually operated within each country, Korea jumps over Japan as the most connected for a large array of cut-off values. What is more, when one tries to get rid of the different $R \& D$ intensities of these 6 
TSs, and work with $\mathbf{R}^{\text {norm }}$ by focusing on the purely relational structure of the TSs, quite surprisingly the picture is even reversed, as Poland now ranks first all along the range.

In front of these appreciable differences, the need of resorting to a sound choice criterion is of course important. Luckily, the same nature of the three network analysis indicators we have used help in inspiring such a choice. As far as density is concerned, its systemic nature would normally suggest the use of $\mathbf{R}^{\text {basket }}$ or $\mathbf{R}^{\text {norm }}$, resorting to the latter when one wants to discount for great differences in countries' aggregate R\&D/GDP ratios. ${ }^{19}$

As far as degree centralization is concerned, the analysis has shown that, in the presence of consistent density gaps across the TSs, the relative TS ranking, both inward and outward, is extremely variable across the range of the relevant threshold values. Accordingly, in this case, instead of comparing the TS centralization at one or more discrete cut-off values, a sounder choice could be to simply compare the absolute and local centralization maximum/minimum of the different TSs, and then checking for which cut-off value they are obtained. In this respect, that is for the sake of this comparison, $\mathbf{R}^{\text {norm }}$ turns out to be the more preferable relativization procedure. Indeed, while it shares with $\mathbf{R}^{\text {basket }}$ the systemic focus of the centralization indicator, it better "centralizes" TS centralization distributions without altering the relevant values. Thus, the main results achieved by using the former method are preserved, while the relative distributions can be more easily compared.

Finally, the conclusion is of course completely different in the case of degree centrality, where the preferred methods should generally be $\mathbf{R}^{\text {unit }}$ and $\mathbf{C}$, because of the main subsystem focus of the analysis based on such indicator.

In concluding the paper, we would like to stress once more that, although mainly intended to address the methodological issues encountered in the comparative analysis of TSs through intersectoral innovation flows matrices, the achieved results turn out to have a broader set of applications. Indeed, they

\footnotetext{
${ }^{19}$ Although possibly less appropriated, the use of $\mathbf{C}$ could be however motivated, as in Leoncini et al. (1996), Leoncini and Montresor (2000a) and Montresor and Vittucci Marzetti (2007b), when one wants to use density as a reference for choosing the cut-off at which then focusing on the different sectors/sub-systems of a TS and on the relative clusters.
} 
can be fruitfully applied each time one deals with value matrices representing input and output flows which have to be somehow relativized in order to remove "scale" effects, whatever they are, and then dichotomized by fixing a certain threshold in order to be able to use the great majority of network analysis tools. The analysis of intersectoral 'knowledge flows' is just an example of this kind of extensions.

\section{References}

Chang, P.-L. and Shih, H.-Y. (2005) Comparing patterns of intersectoral innovation diffusion in Taiwan and China: A network analysis, Technovation, 25(2), pp. 155-169.

DeBresson, C. (1996a) The inter-industrial analysis of innovative activities, in: C. DeBresson (Ed.) Economic Interdependence and Innovative Activity. An Input-Output Analysis (Cheltenham: Edward Elgar), pp. 66-78.

DeBresson, C. (1996b) Why innovative activities cluster, in: C. DeBresson (Ed.) Economic Interdependence and Innovative Activity. An Input-Output Analysis (Cheltenham: Edward Elgar), pp. 149-165.

Dougherty, S. M., Inklaar, R., McGuckin, R. H. and van Ark, B. (2007) International Comparisons of RED Expenditure: Does an RED PPP Make a Difference?, Working Paper 12829, National Bureau of Economic Research, Cambridge, MA.

Edquist, C. (Ed.) (1997) Systems of Innovation. Technologies, Institutions and Organisations (Londra: Pinter Publishers).

Edquist, C. and McKelvey, M. (Eds.) (2000) Systems of Innovation: Growth, Competitiveness and Employment (Cheltenham, UK: Edward Elgar Publishing).

Leoncini, R. (1998) The nature of long-run technological change: innovation, evolution and technological systems, Research Policy, 27, pp. 75-93. 
Leoncini, R., Maggioni, A. and Montresor, S. (1996) Intersectoral innovation flows and national technological systems: network analysis for comparing italy and germany, Research Policy, 25, pp. 415-430.

Leoncini, R. and Montresor, S. (2000a) Classifying technological systems: an empirical application to eight OECD countries, in: P. Saviotti and N. B. (Eds.) Technology and Knowledge: from the Firm to Innovation Systems (Cheltenham: Edward Elgar), pp. 152-173.

Leoncini, R. and Montresor, S. (2000b) Network analysis of eight technological systems, International Review of Applied Economics, 14(2), pp. 213-234.

Leoncini, R. and Montresor, S. (2003a) The technological system, in: R. Leoncini and S. Montresor (Eds.) Technological Systems and Intersectoral Innovation Flows (Cheltenham: Edward Elgar), pp. 33-49.

Leoncini, R. and Montresor, S. (2003b) Technological Systems and Intersectoral Innovation Flows (Cheltenham: Edward Elgar).

Leoncini, R. and Montresor, S. (2005) Accounting for core and extra-core relationships in technological systems: a methodological proposal, Research Policy, 34, pp. 83-100.

Lundvall, B.-A. (Ed.) (1992) National Systems of Innovation. Towards a Theory of Innovation and Interactive Learning (London: Pinter).

Malerba, F. (Ed.) (2004) Sectoral Systems of Innovation: Concepts, Issues and Analyses of Six Major Sectors in Europe (Cambridge: Cambridge University Press).

Marengo, L. and Sterlacchini, A. (1990) Intersectoral technology flows. Methodological aspects and empirical applications, Metroeconomica, 41, pp. 19-39.

Montresor, S. and Vittucci Marzetti, G. (2007a) The deindustrialisation/tertiarisation hypothesis reconsidered: a subsystem application to the OECD7, mimeo. 
Montresor, S. and Vittucci Marzetti, G. (2007b) Innovation clusters in technological systems: a network analysis of 15 OECD countries for the middle '90s, paper presented at the DRUID Conference 2007 (Copenhagen).

OECD (2003) OECD Science, Technology and Industry Scoreboard 2003 Towards a knowledge-based economy (Paris: OECD).

Rampa, G. (1982) Commento a Momigliano e Siniscalco, Moneta e Credito, 139 , pp. $475-479$.

Siniscalco, D. (1982) Il sistema produttivo: Analisi per industrie e subsistemi, Ricerche Economiche, XXXVI(4), pp. 475-488. 


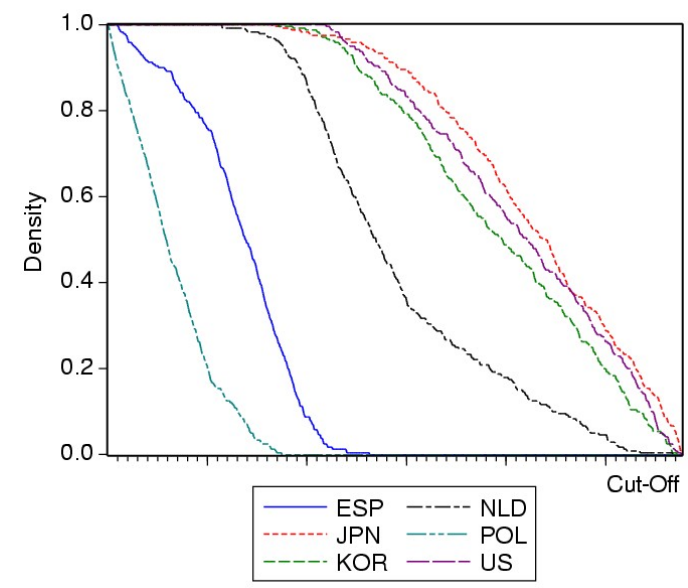

(a) Unit value matrices

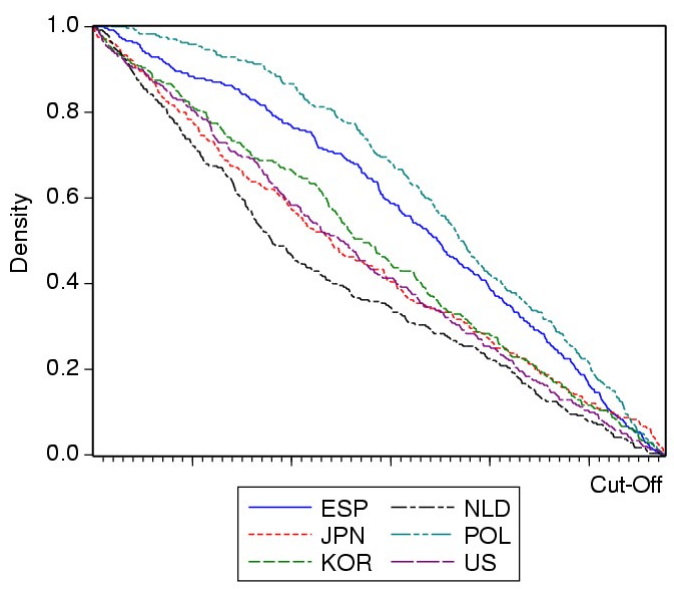

(c) Normalized $\mathbf{R}$

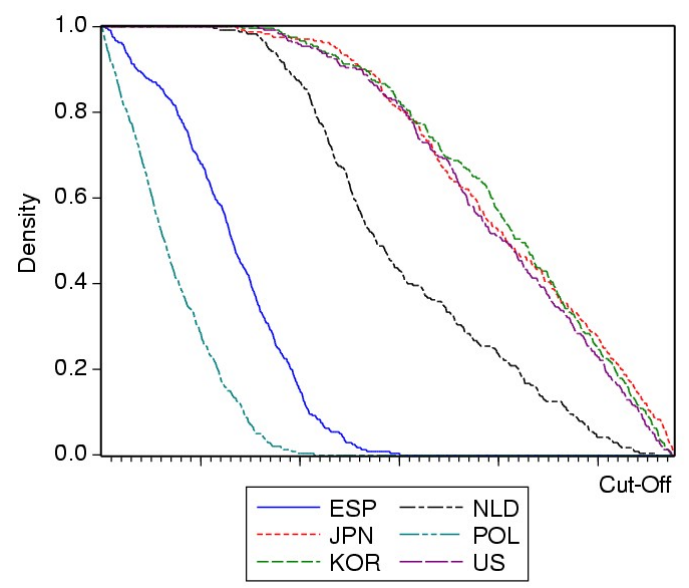

(b) Unit basket of final demand matrices

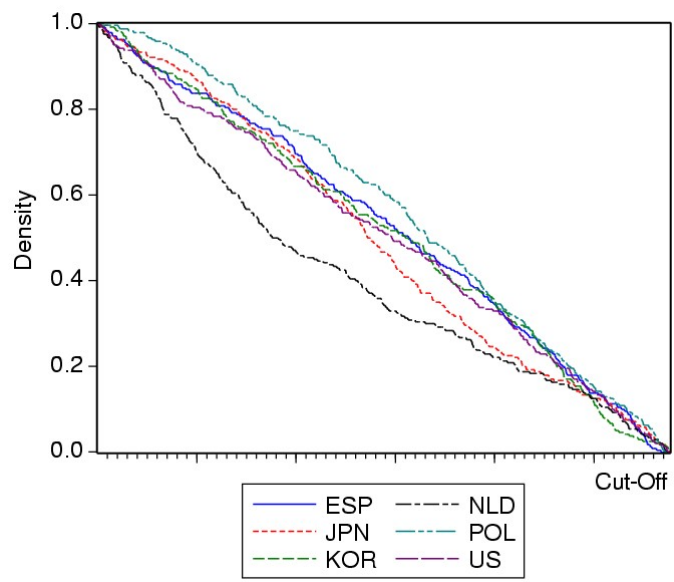

(d) Matrices $\mathbf{C}$

Figure 1: Density distributions for the different relativization methods 


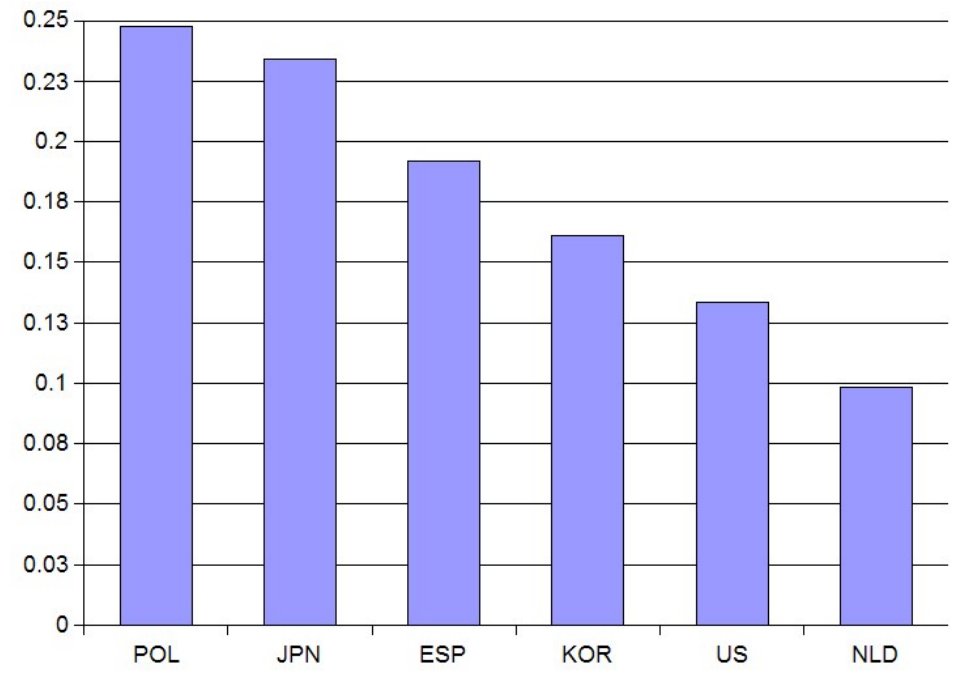

Figure 2: Weight of intersectoral flows on total embodied innovation flows

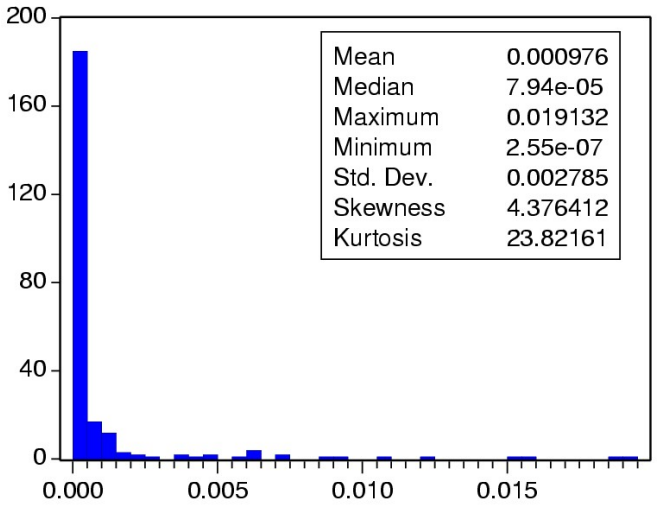

(a) Japan

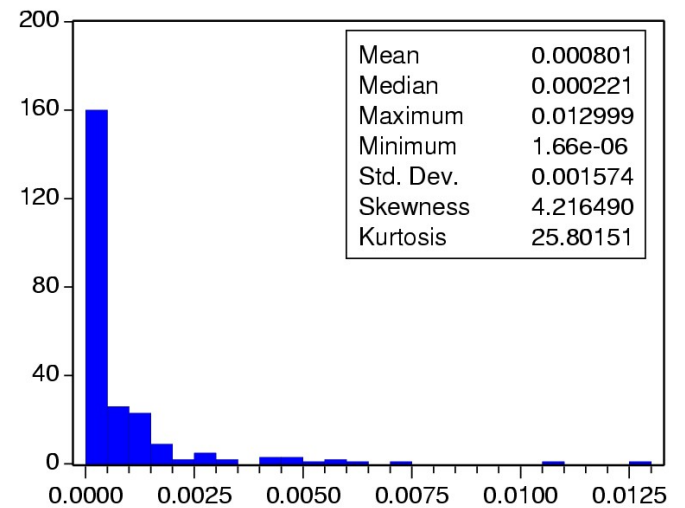

(b) Spain

Figure 3: Distributions of intersectoral flows in normalized $\mathbf{R}$ 


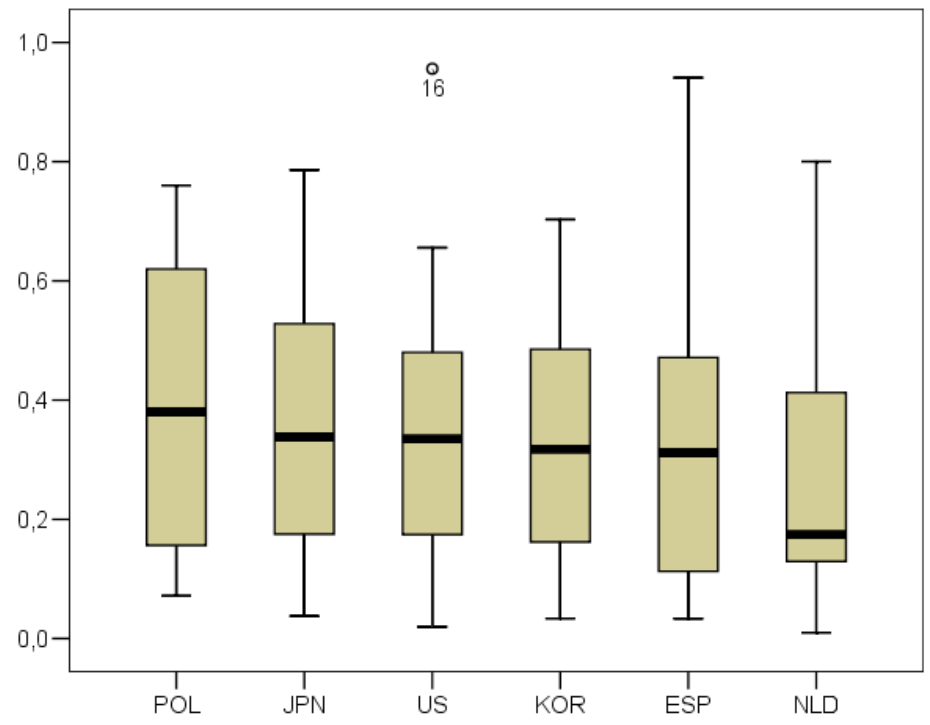

Figure 4: Distributions of the weights of intersectoral innovative acquisitions on total subsystem acquisitions 


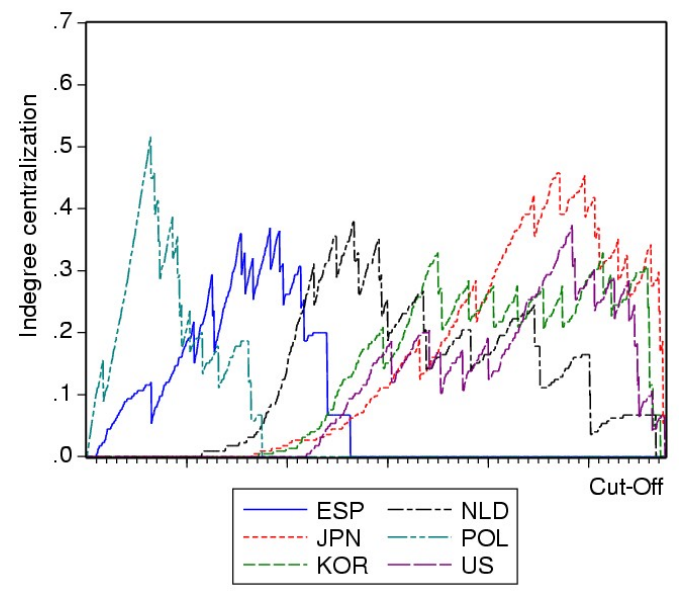

(a) Unit value matrices

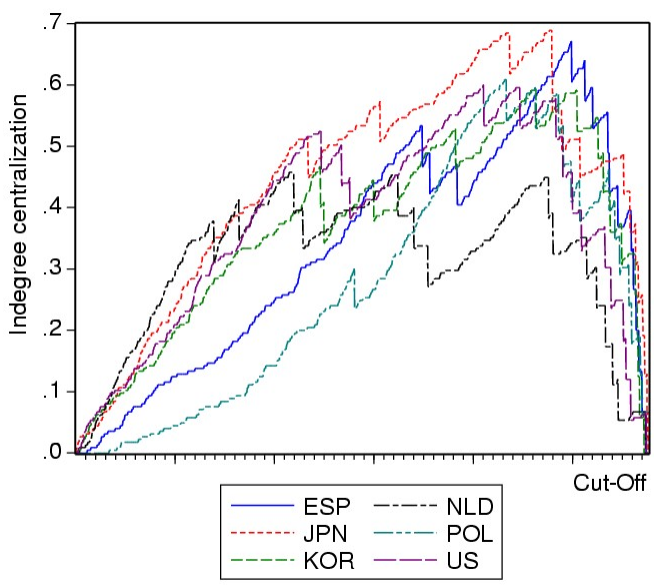

(c) Normalized $\mathbf{R}$

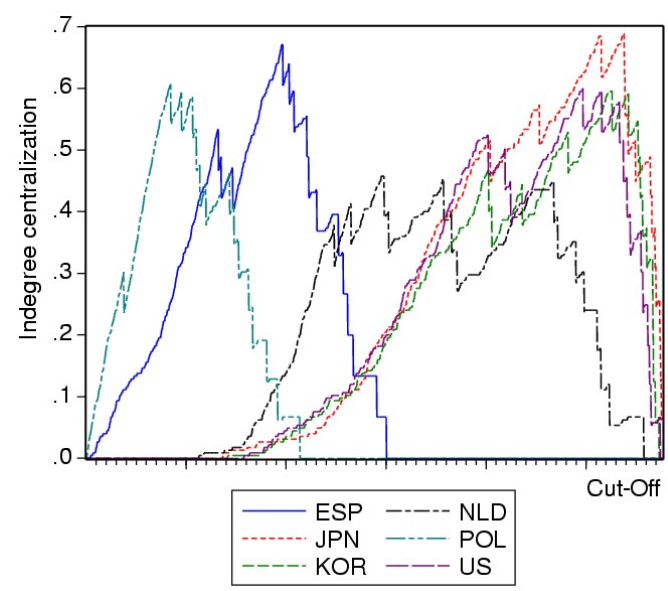

(b) Unit basket of final demand matrices

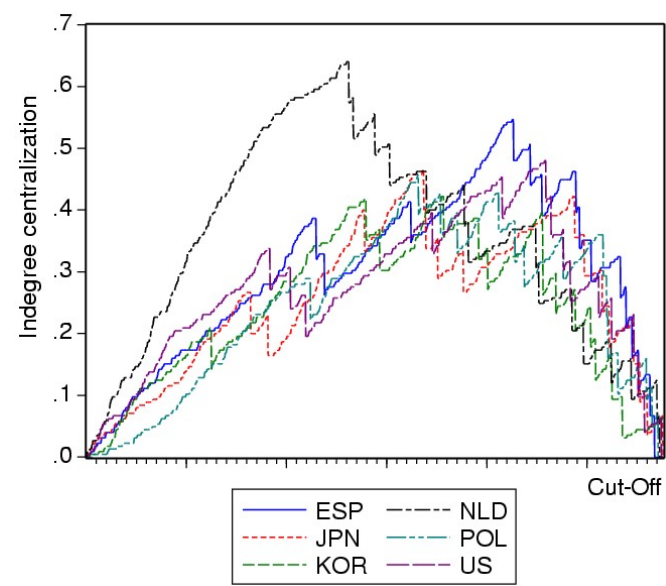

(d) Matrices $\mathbf{C}$

Figure 5: Indegree centralization distributions for the different relativization methods 


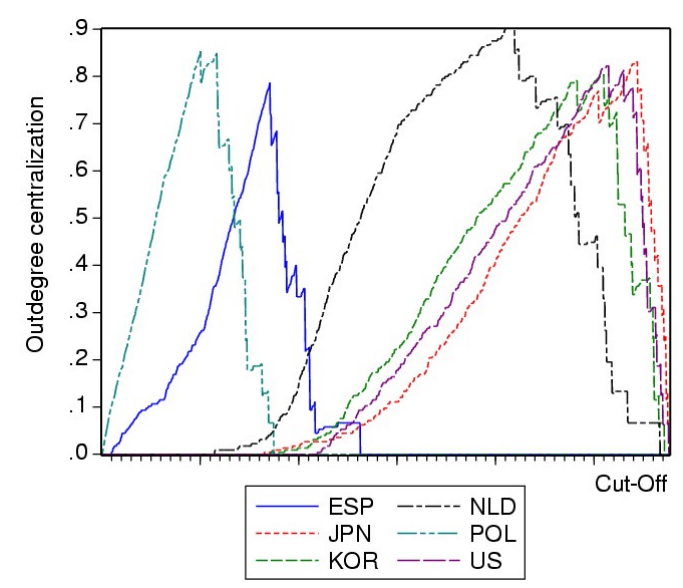

(a) Unit value matrices

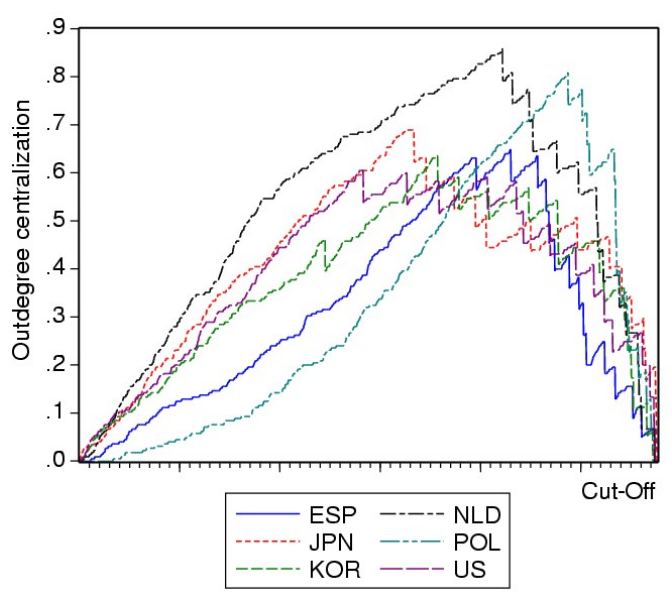

(c) Normalized $\mathbf{R}$

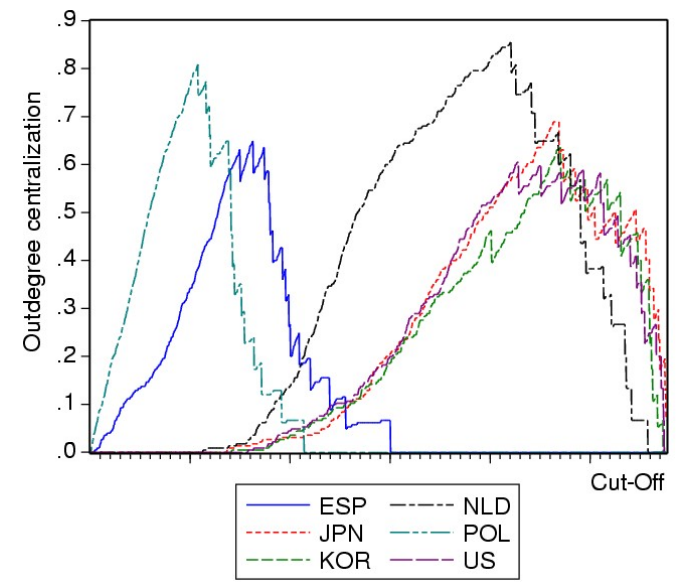

(b) Unit basket of final demand matrices

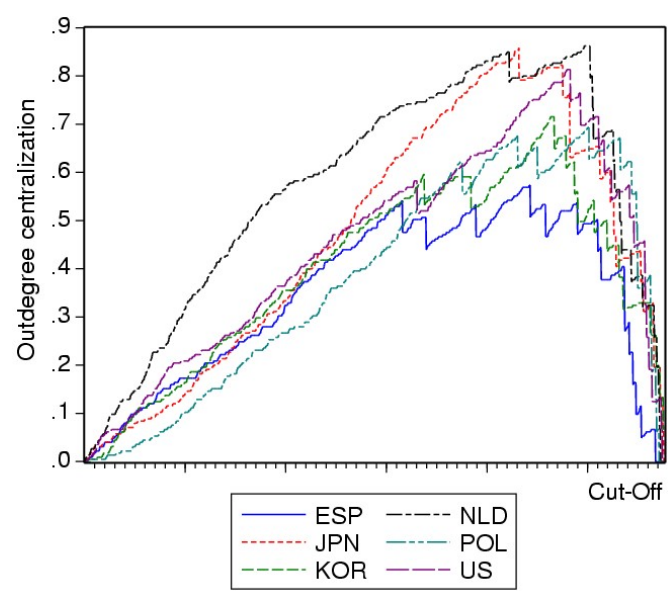

(d) Matrices $\mathbf{C}$

Figure 6: Outdegree centralization distributions for the different relativization methods 


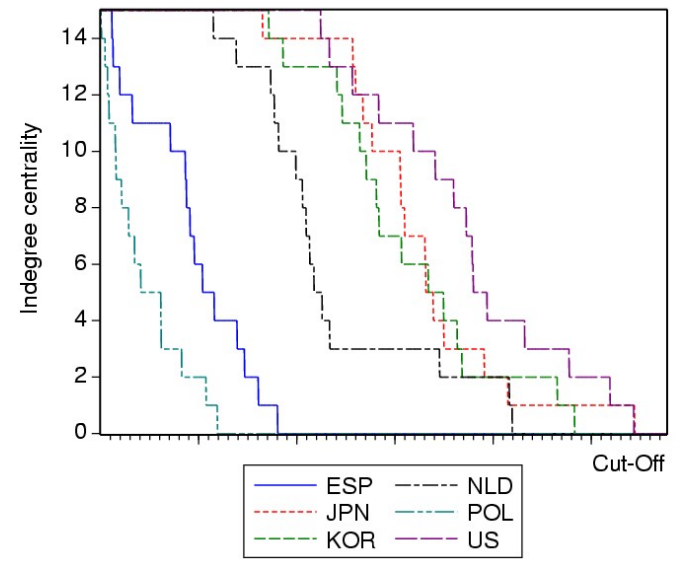

(a) Unit value matrices

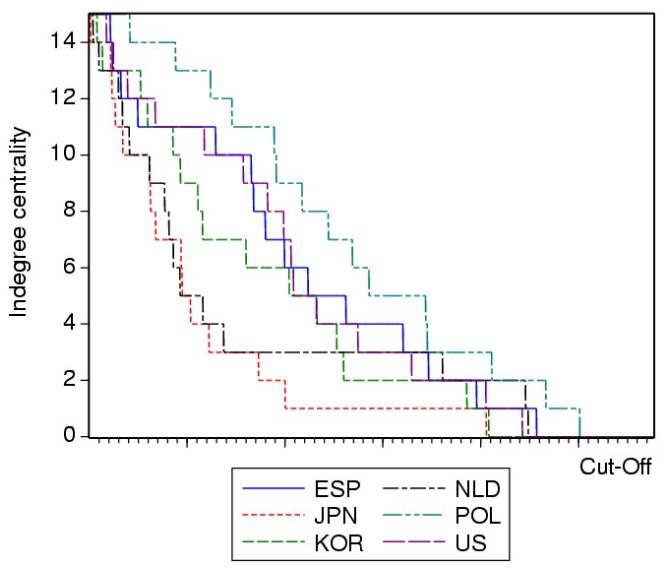

(c) Normalized $\mathbf{R}$

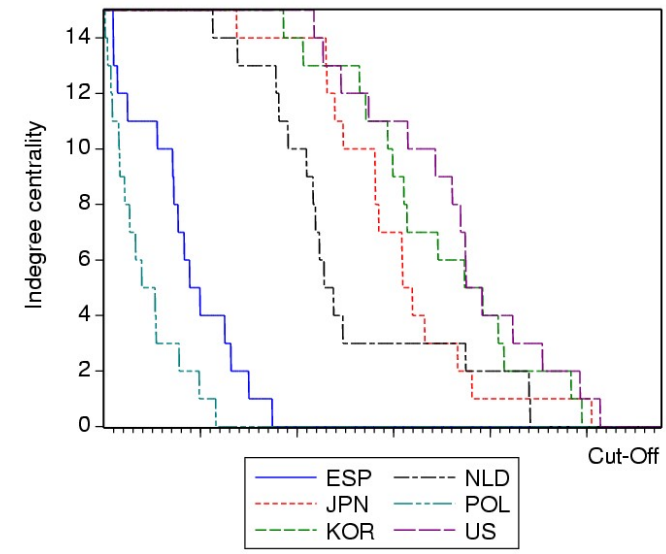

(b) Unit basket of final demand matrices

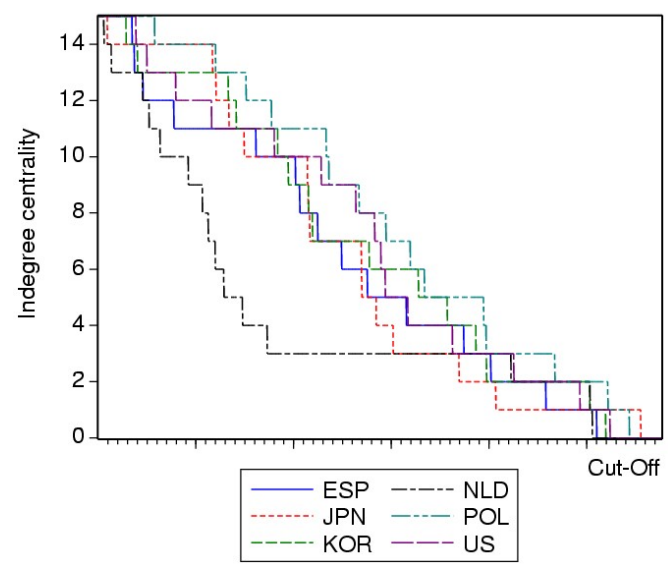

(d) Matrices $\mathbf{C}$

Figure 7: Indegree centrality distributions of textiles for the different relativization methods 


\section{A Sector classification}

\begin{tabular}{clc}
\hline & Sector & ISIC Rev.3 Code \\
\hline 1 & Food products, beverages and tobacco & $15-16$ \\
2 & Textiles, textile products, leather and footwear & $17-19$ \\
3 & Wood, paper, printing, publishing & $20-22$ \\
4 & Coke, refined petroleum products and nuclear fuel & 23 \\
5 & Chemicals (including pharmaceuticals) & 24 \\
6 & Rubber and plastics products & 25 \\
7 & Other non-metallic mineral products & 26 \\
8 & Basic metals & 27 \\
9 & Fabricated metal products (except machinery and equipment) & 28 \\
10 & Machinery and equipment, nec & 29 \\
11 & Electrical and optical instruments & $30-33$ \\
12 & Motor vehicles, trailers and semitrailers & 34 \\
13 & Other transport equipment & 35 \\
14 & Manufacturing, nec; recycling & $36-37$ \\
15 & Electricity, gas and water supply & $40-41$ \\
16 & Construction & 45 \\
\hline
\end{tabular}

\section{B Country coverage}

\begin{tabular}{lc}
\hline Country & I-O and ANBERD data \\
\hline Japan & 1995 \\
Korea & 1995 \\
Netherlands & 1995 \\
Poland & 1995 \\
United States & 1997 \\
\hline
\end{tabular}

